\title{
Estratégias para Elaboração de Game Design de Jogos Digitais Educativos: uma Revisão Sistemática
}

\author{
Kleber Tavares Fernandes ${ }^{1,2}$, Eduardo Henrique da Silva Aranha ${ }^{2}$, \\ Márcia Jacyntha Nunes Rodrigues Lucena ${ }^{1}$ \\ ${ }^{1}$ Departamento de Matemática e Informática Aplicada - DIMAP \\ Programa de Pós-Graduação em Sistemas e Computação - PPgSC \\ Universidade Federal do Rio Grande do Norte - UFRN \\ Caixa Postal 1524 -Lagoa Nova - CEP 59072-970 - Natal/RN - Brasil \\ ${ }^{2}$ Departamento de Ciências Exatas e Tecnologia da Informação \\ Universidade Federal Rural do Semiárido - UFERSA \\ CEP 59515-000 - Campus Angicos/RN - Brasil \\ Kleber76@gmail.com, eduardoaranha@dimap.ufrn.br, marciaj@dimap.ufrn.br
}

\begin{abstract}
The production of educational games is an activity that motivates students to learn the various curricular contents involved in this process. However, creating games requires a meaningful learning curve for nondevelopers, which can be a major challenge for both teachers and students. An effective educational game is certainly the result of designing a well-crafted game design. Thus, it is significant to choose technical and pedagogical strategies that support the elaboration of the game's game design. This article presents a systematic review of the literature on the strategies used in the game design of educational games. Results show that of the 40 primary studies, 16 were included in this study and only 03 present some specific strategy for the development of educational games.
\end{abstract}

Resumo. A produção de jogos educativos é uma atividade que motiva os alunos a aprenderem os diversos conteúdos curriculares envolvidos nesse processo. Porém, criar jogos exige uma curva de aprendizado significativa para não desenvolvedores o que pode ser um grande desafio tanto para professores como para alunos. Um jogo educativo eficaz certamente é resultado da concepção de um game design bem elaborado. Assim, é significativo escolher estratégias técnicas e pedagógicas que deem suporte a elaboração do game design do jogo. Esse artigo apresenta uma revisão sistemática da literatura a respeito das estratégias utilizadas na concepção de game design de jogos educativos. Os resultados mostram que dos 40 estudos primários, 16 foram incluídos nesse trabalho e apenas 03 apresentam alguma estratégia especifica para o desenvolvimento de jogos educativos.

\section{Introdução}

O uso de jogos digitais na educação se tornou uma importante estratégia de ensinoaprendizagem. Esses recursos já fazem parte do cotidiano e do desenvolvimento de 
VII Congresso Brasileiro de Informática na Educação (CBIE 2018)

Anais do XXIX Simpósio Brasileiro de Informática na Educação (SBIE 2018)

muitas crianças, jovens e adultos, oferecendo muito mais do que apenas entretenimento. Os jogos digitais em geral promovem um maior engajamento do jogador, despertando sua curiosidade e ampliando as possibilidades de aprendizagem. Esses são alguns motivos que levaram muitos educadores a perceberem o potencial dos jogos digitais para fins educacionais [Rebouças et. al. 2010], [Marques et. al. 2011], [Sá et. al. 2007], [Prensky 2010].

Muitos estudos defendem não apenas o uso nas escolas, mas também o desenvolvimento de jogos digitais por alunos como estratégia de aprendizagem [Marinho et. al. 2011]. Gomes (2014) e Sanford (2007) enfatizam em suas pesquisas os ganhos de aprendizagem, desenvolvimento da autonomia e criatividade quando jogos são desenvolvidos em sala de aula. Dar oportunidade para alunos criarem seus próprios jogos favorece o seu engajamento, a sua motivação, o seu desenvolvimento cognitivo e a aprendizagem dos conteúdos curriculares envolvidos nesse processo [Danielle et. al. 2015].

No entanto, desenvolver jogos digitais é uma atividade bastante complexa que envolve conhecimentos específicos e equipes multidisciplinares. $\mathrm{O}$ processo de produção de um jogo inicia com a definição do seu conceito inicial (Game Design) e termina com a criação de uma versão final do jogo, sendo que várias etapas acontecem entre esses dois pontos [Chandler 2012].

Segundo Tang (2008), os métodos de desenvolvimento de jogos digitais são os mesmos da engenharia de software. Além disso, as tecnologias utilizadas no desenvolvimento de jogos digitais educacionais são similares às dos jogos de entretenimento convencionais. Dessa forma, criar jogos exige uma curva de aprendizado significativa para quem não tem formação em computação, sendo assim um grande desafio tanto para professores como para alunos do ensino básico. Porém, no contexto de jogos educativos desenvolvidos nas escolas, esse problema é reduzido utilizando-se ferramentas mais simples de programação visual, como Scratch, Stencyl, RPG Maker, Construct 3 , entre outras.

Entretanto, para se obter um jogo educacional interessante, é necessário ter um game design bem elaborado, que trabalhe tanto aspectos de entretenimento como educacionais. De fato, a escolha de estratégias eficientes para suportar a elaboração do game design de jogos no contexto educacional mostra-se determinante e pode reduzir a barreira da adoção da aprendizagem baseada em jogos. Sendo assim, é importante verificar na literatura as estratégias propostas, seus benefícios e limitações.

Desta forma, este artigo apresenta numa Revisão Sistemática da Literatura (RSL) que investiga estratégias (abordagens, modelos, métodos, linguagens e/ou experiências) para elaboração de game design de jogos digitais no contexto educacional. Foram extraídos, catalogados, analisados e sintetizados dados de vários estudos primários publicados em congressos, jornais e periódicos nacionais, que abordam a temática desta revisão. Os resultados desta pesquisa servem de parâmetro para a identificação de estratégias que podem ser utilizadas no desenvolvimento de jogos digitais por professores e alunos em sala de aula, bem como servindo de base para $\mathrm{o}$ desenvolvimento de novas abordagens de game design de jogos educativos. 
VII Congresso Brasileiro de Informática na Educação (CBIE 2018)

Anais do XXIX Simpósio Brasileiro de Informática na Educação (SBIE 2018)

Este artigo está organizado em mais cinco seções além desta introdução. A Seção 2 apresenta o método de pesquisa utilizado. Já a Seção 3 mostra a análise dos dados e a Seção 4 as ameaças a validade deste estudo. Por fim, a Seção 5 apresenta as considerações finais.

\section{Metodologia}

Este trabalho tem por objetivo investigar, no contexto nacional, trabalhos científicos que apresentem estratégias utilizadas na concepção de game design de jogos educativos, no período de 2010 a 31 de dezembro de 2017. A metodologia utilizada neste trabalho foi baseada nas diretrizes propostas por Kitchenham et. al. (2009). Segundo a autora, uma RSL é uma pesquisa em profundidade de um fenômeno de interesse que produz resultados específicos e detalhados por meio da análise de conteúdo e qualidade do material pesquisado. Para esta revisão, as questões de pesquisa que guiaram todo o trabalho são apresentadas na Tabela 1.

Tabela 1: Questões de Pesquisa

\begin{tabular}{|c|l|}
\hline Questões & \multicolumn{1}{|c|}{ Descrição } \\
\hline QP1 & Quais as estratégias utilizadas na elaboração do game design de jogos educativos? \\
\hline QP2 & Qual o perfil de quem produz o game design dos jogos? \\
\hline QP3 & Qual o público alvo dos game designs propostos? \\
\hline QP4 & Como as abordagens propostas estão sendo aplicadas e/ou validadas? \\
\hline QP5 & As abordagens sendo propostas são inspiradas em alguma outra? \\
\hline QP6 & Quais são os benefícios apresentados pelas abordagens sendo propostas? \\
\hline QP7 & Quais são as limitações apresentadas pelas abordagens sendo propostas? \\
\hline
\end{tabular}

A obtenção dos estudos primários foi realizada através de uma string de busca para trazer trabalhos que tratassem de estratégias de game design, sendo por isso formulada com as palavras-chave linguagem, modelo, metodologia, abordagem e game design. Optamos por não colocar a palavra "educação", nesse primeiro momento, para ampliarmos o número de artigos resultantes da busca e observarmos o que ainda não está sendo aplicado na área de game design para jogos educativos.

O procedimento utilizado para busca dos estudos primários compreendeu: (i) utilizar os sistemas de busca dos anais do Simpósio Brasileiro de Informática na Educação (SBIE), Revista Brasileira de Informática na Educação (RBIE) e Workshop de Informática na Escola (WIE); e (ii) realizar busca manual nos anais do Simpósio Brasileiro de Computação, Jogos e Entretenimento Digital (SBGAMES), que não possuía no momento da pesquisa um sistema de busca próprio.

Os artigos encontrados foram então analisados segundo os critérios de inclusão (CI) e critérios de exclusão (CE) mostrados nas Tabelas 2 e 3. Essa análise foi realizada inicialmente pelo título, resumo e palavras chave. Em seguida pelas seções de introdução e conclusão. E finalmente pela leitura completa dos artigos. 
VII Congresso Brasileiro de Informática na Educação (CBIE 2018)

Anais do XXIX Simpósio Brasileiro de Informática na Educação (SBIE 2018)

Tabela 2: Critérios de Inclusão

\begin{tabular}{|l|l|}
\hline Critérios & \multicolumn{1}{c|}{ Descrição } \\
\hline CI1 & $\begin{array}{l}\text { Estudos que apresentem estratégias (linguagens, modelos, metodologias e/ou experiências) } \\
\text { utilizadas na concepção do game design de jogos sérios. }\end{array}$ \\
\hline CI2 & Trabalhos nas línguas portuguesa, espanhola e inglesa. \\
\hline CI3 & Trabalhos publicados em conferências e jornais. \\
\hline
\end{tabular}

Tabela 3: Critérios de Exclusão

\begin{tabular}{|l|l|}
\hline Critérios & \multicolumn{1}{|c|}{ Descrição } \\
\hline CE1 & $\begin{array}{l}\text { Estudos semelhantes que apresentam a evolução de uma mesma pesquisa e que contenham } \\
\text { os mesmos autores. (Excluir as versões mais antigas.) }\end{array}$ \\
\hline CE2 & Trabalhos fora do período da pesquisa. \\
\hline CE3 & Trabalhos cujos textos completos não estejam disponíveis para acesso gratuito na web. \\
\hline CE4 & $\begin{array}{l}\text { Documentos que estão disponíveis na forma de resumos, apresentações ou estudos } \\
\text { secundários (revisões sistemáticas da literatura, mapeamentos sistemáticos, etc.). }\end{array}$ \\
\hline
\end{tabular}

A qualidade dos estudos encontrados também foi avaliada através dos cinco critérios apresentados na Tabela 4. Esses critérios foram utilizados para determinar o rigor, a análise e a credibilidade dos métodos de pesquisa empregados, bem como a relevância e a qualidade de cada estudo para esta revisão. As respostas para cada questão foram tabuladas e atribuídas um valor de 1 ("Sim"), 0,5 ("parcialmente") ou 0 ("Não").

Tabela 4: Avaliação da Qualidade

\begin{tabular}{|l|l|}
\hline Avaliação & \multicolumn{1}{|c|}{ Descrição } \\
\hline AV1 & Existe uma definição clara dos objetivos da pesquisa? \\
\hline AV2 & Existe uma descrição adequada do contexto em que a pesquisa foi realizada? \\
\hline AV3 & Existe clareza na estratégia descrita na pesquisa? \\
\hline AV4 & Há um processo de avaliação da estratégia descrita na pesquisa? \\
\hline AV5 & O estudo apresenta uma comparação com outras estratégias existentes? \\
\hline
\end{tabular}

Com o objetivo de testarmos a validade do procedimento de avaliação e a qualidade dos estudos, envolvemos um segundo e terceiro revisor. Foram distribuídas as mesmas amostras dos estudos e aplicados os mesmos critérios de avaliação. A avaliação da qualidade manteve-se coerente entre os participantes desse processo.

Por fim, foram extraídos os seguintes dados a partir dos estudos primários selecionados: dados de identificação do estudo, objetivos, método utilizado, perfil dos participantes, critérios de avaliação da qualidade, resultados, conclusões e dificuldades apresentadas. Esses dados foram coletados por apenas um revisor, enquanto que os demais analisaram o resultado geral dos estudos. Comparado os resultados, percebemos que não existiu incoerência na estratégia de extração dos dados. 
VII Congresso Brasileiro de Informática na Educação (CBIE 2018)

Anais do XXIX Simpósio Brasileiro de Informática na Educação (SBIE 2018)

\section{Análise dos Artigos Encontrados}

A Tabela 5 apresenta a string de busca utilizada, bem como a quantidade de artigos retornados por cada uma das fontes de artigos acessadas. Os resultados detalhados deste processo de busca podem ser visualizados com mais detalhes através do link https://goo.gl/vEuBfF.

Tabela 5: String de Busca

\begin{tabular}{|c|c|c|c|c|}
\hline \multirow{2}{*}{ String de busca } & \multicolumn{4}{|c|}{ Fonte de Dados } \\
\cline { 2 - 5 } $\begin{array}{c}\text { ((linguagem OR modelo OR metodologia OR abordagem) AND } \\
\text { game design) }\end{array}$ & 1 & 2 & 7 & 30 \\
\hline
\end{tabular}

Todos os estudos primários recuperados foram analisados com o objetivo de selecionar aqueles que contribuem para responder as questões de pesquisa. Analisamos inicialmente 40 estudos, com a leitura dos títulos, palavras chaves e resumos. Permaneceram selecionados 22 estudos. Desse total, após leitura das principais seções (introdução, conclusão e elementos gráficos) e nova aplicação dos critérios de análise, restaram 19 estudos para leitura completa. Essa última etapa resultou na exclusão de mais 3 artigos, finalizando o processo com 16 estudos para extração e análise dos dados para responder as questões de pesquisa apresentadas.

A Tabela 6 apresenta os resultados da avaliação da qualidade dos estudos selecionados. Todos os artigos possuem definição clara dos seus objetivos (AV1), o que facilitou o entendimento das suas propostas (AV3). Observamos que apenas 9 estudos apresentam uma descrição adequada do contexto em que a pesquisa foi realizada (AV2). Dos 16 estudos, 7 não mostram um processo de avaliação da abordagem proposta bem definido, o que compromete a sua efetiva aplicação (AV4). Por fim, somente 4 estudos apresentam uma comparação com outras abordagens existentes (AV5).

Tabela 6: Resultados da Avaliação da Qualidade

\begin{tabular}{|c|c|c|c|c|c|c|}
\hline ESTUDO & AV1 & AV2 & AV3 & AV4 & AV5 & TOTAL \\
\hline E1 & 1 & 1 & 1 & 1 & 0 & 4 \\
\hline E2 & 1 & 1 & 1 & 1 & 0 & 4 \\
\hline E3 & 1 & 1 & 1 & 1 & 0 & 4 \\
\hline E4 & 1 & 1 & 1 & 0 & 0 & 3 \\
\hline E5 & 1 & 0 & 1 & 0 & 0 & 2 \\
\hline E6 & 1 & 1 & 1 & 1 & 1 & 5 \\
\hline E7 & 1 & 0 & 1 & 0 & 0 & 2 \\
\hline E8 & 1 & 0 & 1 & 1 & 0 & 3 \\
\hline E9 & 1 & 1 & 1 & 1 & 0 & 4 \\
\hline E10 & 1 & 1 & 1 & 1 & 0 & 4 \\
\hline E11 & 1 & 0 & 1 & 0 & 1 & 3 \\
\hline E12 & 1 & 1 & 1 & 1 & 0 & 4 \\
\hline E13 & 1 & 0 & 1 & 0 & 0 & 2 \\
\hline E14 & 1 & 0 & 1 & 0 & 1 & 3 \\
\hline
\end{tabular}


VII Congresso Brasileiro de Informática na Educação (CBIE 2018)

Anais do XXIX Simpósio Brasileiro de Informática na Educação (SBIE 2018)

\begin{tabular}{|l|l|l|l|l|l|l|}
$\mathrm{E} 15$ & 1 & 0 & 1 & 0 & 0 & 2 \\
\hline $\mathrm{E} 16$ & 1 & 1 & 1 & 1 & 1 & 5 \\
\hline
\end{tabular}

Dois dos estudos obtiveram a pontuação máxima de 05 pontos [E6 e E16]. A pontuação mais baixa que os artigos alcançaram foi de 2 pontos [E5, E7, E13 e E15]. A pontuação média em qualidade dos trabalhos incluídos na revisão é de 3.38 pontos numa escala de 0 a 5 pontos. O critério AV5 foi o menos atendido, indicando uma ausência de estudos comparativos em boa parte dos trabalhos encontrados.

A seguir, apresentamos as respostas às questões de pesquisa da revisão sistemática obtidas a partir da análise dos artigos encontrados.

\section{QP1 - Quais as estratégias utilizadas na elaboração do game design de jogos educativos?}

A primeira questão de pesquisa deste trabalho objetivou identificar as estratégias (abordagens, linguagens, modelos, metodologias e/ou experiências) propostas para a concepção do game design de jogos educativos.

Os resultados apontam uma variedade de 15 propostas diferentes: prototipagem [E1 e E6], descrição textual [E2], Scrum4Games [E4], design participativo [E5], Game Componentes Framework [E6], RhetorGames [E7], Game Design Patterns [E8], Blue Ocean Strategy and Personas [E9], Game design thinging [E10], Game Design Document - GDD[E11 e E13], ViP [12], Unified Game Canvas [E15], Interativa e integradora [E16], Design, Play and Experience (DPE) e The learning games design model [E14]. Apenas duas técnicas (prototipagem e GDD) apareceram em mais de um trabalho. Todas são propostas isoladas baseadas ou não em outras estratégias, como veremos mais a seguir.

\section{QP2 - Qual o perfil de quem produz o game design dos jogos?}

Quando observamos o perfil de quem produz o game design dos jogos nos estudos selecionados, game designers aparecem na maioria dos trabalhos. Entretanto, conforme ilustrado na Figura 1a, alguns trabalhos envolvem: estudantes de nível superior ou pósgraduação [E1 e E10], estudantes e profissionais da área de jogos [E2 e E12], game designes e desenvolvedores [E3, E4 e E16], apenas game designers [E6. E7, E8, E11, E14 e E15] e equipe multidisciplinar [E9 e E13]. Um dos estudos não especificou o perfil de quem produz o game design dos jogos [E5].

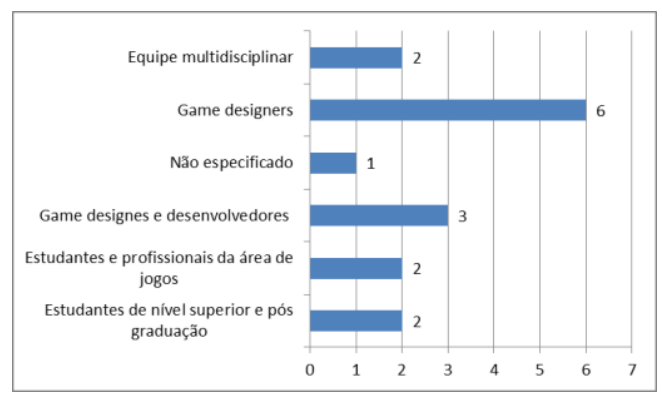

Figura 1a: Perfil de quem produz os game designs.

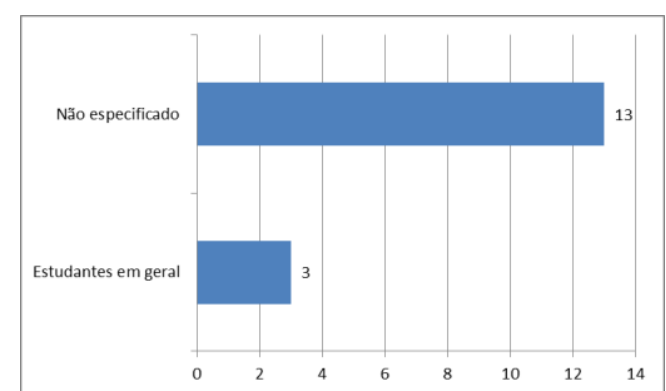

Figura 1b: Perfil do público alvo dos jogos. 
VII Congresso Brasileiro de Informática na Educação (CBIE 2018)

Anais do XXIX Simpósio Brasileiro de Informática na Educação (SBIE 2018)

\section{QP3 - Qual o público alvo dos game designs propostos?}

Em relação ao público alvo, verificamos que a maioria dos game designs desenvolvidos pelos trabalhos analisados não especifica público alvo. Apenas três estudos definiram o perfil como estudantes em geral [E10, E14 e E16], conforme visto na Figura 1b.

Esse resultado mostra evidências de que não há uma preocupação em saber qual o perfil do jogador para qual o game design do jogo será desenvolvido, o que pode comprometer a eficiência do produto final.

\section{QP4 - Como as abordagens propostas estão sendo aplicadas e/ou validadas?}

Outra informação bastante importante extraída dos resultados da análise dos estudos é que $63 \%$ das estratégias foram aplicadas e validadas [E1, E2, E3, E4, E6, E8. E9, E10, E12, E15]. Isso demonstra que os estudos não ficaram apenas no plano das ideias e provas de conceito, mas passaram por algum processo de experimentação. Percebemos ainda, através da analise dos resultados dos estudos, que a maioria dos trabalhos realiza a aplicação da proposta em cenários simples, necessitando de estudos adicionais para melhor validação das propostas. Além disso, os autores dos trabalhos que não foram validados reconhecem nos artigos que os mesmos necessitam passar por essa etapa [E5, E11, E13, E14 e E16]. A Figura 2a apresenta essas informações de forma gráfica.

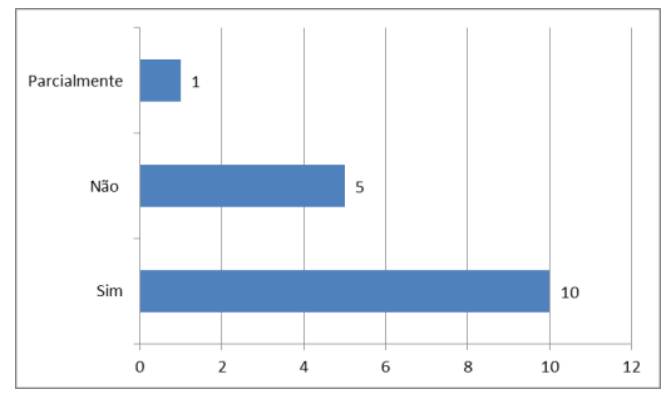

Figura 2a: Validação das propostas.

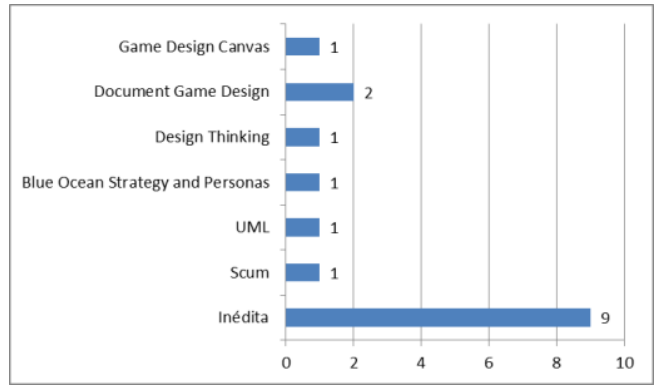

Figura 2b: Estudos inspirados em outras abordagens.

\section{QP5 - As abordagens sendo propostas são inspiradas em alguma outra?}

A análise dos artigos encontrados aponta que as abordagens propostas por esses estudos advêm de métodos bastante reconhecidos na academia, tais como: Scrum [E4], UML [E6], Design Thinking [E10], Document Game Design [E11] e [E13], Blue Ocean Strategy and Personas [E9], e Game Design Canvas [E15]. Todos os demais não foram inspirados em outras estratégias, conforme ilustrado na Figura $2 b$.

\section{QP6 - Quais são os benefícios apresentados pelas abordagens sendo propostas?}

Após a análise de todos os estudos incluídos na revisão sistemática, foi possível extrair os principais benefícios de cada estratégia reportados pelos seus autores que, de forma resumida, são apresentados a seguir: "Early Game Design Rehearsal with Paper Prototyping" [E1] - A abordagem permite a apreciação das qualidades, timing, complexidade e interesse pelo jogo. Utiliza papel não dependendo de recursos tecnológicos; "Estudo de Formatos Alternativos para Documentação de Game Design" 
VII Congresso Brasileiro de Informática na Educação (CBIE 2018)

Anais do XXIX Simpósio Brasileiro de Informática na Educação (SBIE 2018)

[E2] - A descrição do jogo através de imagens apresentou melhor clareza na definição dos requisitos e funcionamento do jogo; "Game Design e Prototipagem: Conceitos e Aplicações ao Longo do Processo Projetual" [E3] - Prototipagem permite construir um jogo mais imersivo e funcional com foco no jogador. Permite melhor visualização da ideia do jogo e melhor comunicação entre a equipe. Oferecem redução de tempo e custo; "Scrum4Games: Uma aplicação do Scrum para projetos de games focada em game design" [E4] - O trabalho decorreu em uma sistematização e organização das diversas funções presentes na produção de um game de acordo com a lógica da metodologia Scrum; "Um modelo de Game Design orientado à participação" [E5] - A possibilidade da participação do usuário no processo de desenvolvimento do jogo; "Towards a Library of Game Components: A Game Design Framework Proposal" [E6] - Reuso dos componentes da biblioteca; "Integrating Rhetoric to Game Design" [E7] - Fácil assimilação; "Towards a Game Design Patterns Suggestion Tool: The documentation of a computerized textual analysis experimente" [E8] - Relaciona padrões de game designs de jogos; "Applying Blue Ocean Strategy to Game Design: A Path to Innovation" [E9] Facilita o uso no contexto dos jogos pela estratégia original ser conhecida na área da administração; "Game thinking is not game design thinking!" [E10] - Melhora a criatividade dos alunos. Propostas mais inovadoras. Melhor visualização das características dos jogos através de canvas; "Criação de um modelo conceitual para documentação de Game Design" [E11] - Proposta de um GDD unificado que possibilite a documentação das suas ideias de maneira clara e sucinta; "Estudo comparativo entre diferentes abordagens de game design" [E12] - As abordagens foram comparadas com resultados equivalentes. A ViP mostrou-se mais adequada ao perfil da indústria; "Simple Game Design Document Focused on Gameplay Features" [E13] - Foco no usuário; "Estudo comparativo entre modelos de game design para jogos educacionais" [E14] Ambos possuem objetivos educacionais claros. Consideram equipes multidisciplinares. Representação simplificada; "Uma Proposta de Game Design Canvas Unificado" [E15] - Reúne os principais elementos de diversos GDC. Modelagem rápida. Cobertura unificada e diferenciada dos fundamentos dos jogos; "Metodologia de Desenvolvimento de Jogos Sérios: especificação de ferramentas de apoio open source" [E16] - Criação de um framework com metodologia e ferramentas para desenvolvimento do jogos.

\section{QP7 - Quais são as limitações apresentadas pelas abordagens sendo propostas?}

Esta pesquisa analisou as principais limitações reportadas para as propostas apresentadas nos artigos analisados. O estudo [E1] limita-se a fazer o game design de projetos iniciais de jogos. A descrição incompleta dos requisitos do jogo e a dificuldade de interpretação de texto foram as limitações do estudo [E2]. Em um caso a prototipação por si só não garantiu o sucesso do jogo [E3]. Os estudos [E4, E5, E7, E10, E11, E1, E14, E15 e E16] apresentam como limitação o processo (ou a falta) de validação por outras equipes de desenvolvimento.

Outras limitações também foram reportadas por outros trabalhos: falta de métricas e indicadores de avaliação da proposta [E16]; modelo cansativo de preencher [E15]; plataforma de distribuição com recursos limitados [E13]; foca em conceitos de alto nível [E10]; falta de maturidade da ferramenta para aplicação na indústria [E8]. 
VII Congresso Brasileiro de Informática na Educação (CBIE 2018)

Anais do XXIX Simpósio Brasileiro de Informática na Educação (SBIE 2018)

\section{Ameaças à Validade}

De forma intrínseca a uma revisão da literatura, as principais ameaças à validade desta revisão sistemática estão relacionadas ao viés de seleção de publicações e a extração de dados imprecisos. Para controlar essa ameaça, este trabalho observou as principais fontes de artigos nacionais apoiadas pela Sociedade Brasileira da Computação na área de educação e de jogos digitais.

Foi utilizada uma string de busca diretamente relacionada aos objetivos e questões formuladas para esta revisão, reduzindo significativamente o risco de artigos de algumas fontes não serem retornados pelo motor de busca utilizado. Desta forma, consideramos que as ameaças a este estudo foram devidamente controladas pelo rigor metodológico utilizado.

\section{Conclusões}

Nessa pesquisa, apresentamos uma revisão sistemática da literatura com o objetivo de conhecer as estratégias (abordagens, modelos, métodos, linguagens e/ou experiências) para elaboração de game design de jogos digitais no contexto educacional. Percebemos que no processo de busca dos estudos primários a palavra chave "educação" reduziria muito o número de trabalhos selecionados. Por isso, optamos por retirá-la e analisarmos os aspectos educacionais nas etapas seguintes da revisão, durante a leitura desses artigos. Os resultados mostram que dos 16 estudos selecionados apenas 3 [E10, E14 e E16] apresentam estratégias de criação de game design de jogos voltados para educação, o que demonstra uma enorme carência nessa área. E mesmo a maioria dos estudos sendo no contexto da indústria, a análise dos mesmos permite refletir sobre as estratégias propostas nesses outros contextos e de como elas podem ser utilizadas ou modificadas para o cenário da educação.

Outro fato que merece atenção é a necessidade de uma sistematização da aplicação, teste e validação das propostas. A maioria apresentou ausência ou superficialidade nesse processo. Sugerimos a realização de um maior número de estudos de casos e ou experimentos, em cenários diferentes, bem como a elaboração de critérios rigorosos para melhor avaliação dos resultados.

Finalmente, os trabalhos analisados indicam que a correta elaboração do game design mostra-se determinante para o desenvolvimento de um jogo digital eficaz. Como observado por esta revisão, esse papel geralmente é realizado pelo game designer. Porém, no contexto educacional, como mostrado por alguns trabalhos, este trabalho pode ser executado por professores e alunos. Essa pode ser uma excelente estratégia de ensino aprendizagem baseada em jogos.

\section{Referências}

Danielle, N. Gomes, Araújo, Luiz, Machado, Thiago, Filho, Angelo, Souza, Silvana. (2015) "Ensino Aprendizagem Através de Desenvolvimento de Jogos". In: Simpósio Brasileiro de Jogos - SBGames 2015. 
VII Congresso Brasileiro de Informática na Educação (CBIE 2018)

Anais do XXIX Simpósio Brasileiro de Informática na Educação (SBIE 2018)

K. R. Gomes; D. L. Batista. (2014) "Designer de games x educadores: discutindo sobre os jogos eletrônicos na formação de professores". In: S. Lucena, (org.) Cultura digital, jogos eletrônicos e educação, p. 99-119. Salvador, EDUFBA, 2014.

K. Sanford; L. Madill. (2007) "Recognizing New Literacies: Teachers and Students Negotiating the Creation of Video Games in School”. Situated Play, Conference Proceedings. Japan, DiGRA, 2007.

Kitchenham, B.; Brereton, P.; Budgen, D.; Turner, M.; Bailey, J.; Linkman, S. (2009) "Systematic literature reviews in software engineering - A systematic literature review". In: Information and Software Technology, v.51, p.7-15.

Kyriacou, M. T.; Brereton, O. P. (2011) "Systematic Literature Review: Teaching Novices Programming Using Robots". In: International Conference on Evaluation and Assessment in Software Engineering - EASE.

Major, L., Kyriacou, T., Brereton, O. P. (2011) "Systematic Literature Review: Teaching Novices Programming Using Robots". In: International Conference on Evaluation and Assessment in Software Engineering - EASE.

Marinho, F. C. V., Giannella, T. R. and Struchiner, M. (2011) "Estudantes do Ensino Básico Como Desenvolvedores de Jogos Digitais: Contextos Autênticos de Aprendizagem para Educação em Ciências e Matemática". In: VIII Encontro Nacional de Pesquisa em Educação em Ciências, Campinas, São Paulo.

Marques, D. L.; Costa, L. F. S.; Silva, M. A. A.; Rebouças, A. D. D. S. (2011) "Atraindo Alunos do Ensino Médio para a Computação: Uma Experiência Prática de Introdução a Programação utilizando Jogos e Python". In: Simpósio Brasileiro de Informática na Educação - SBIE, Aracaju - SE.

Prensky, M. (2010) "Não me atrapalhe, mãe - eu estou aprendendo". São Paulo: Phorte.2010.

Rebouças, A. D. D. S.; Marques, D. L.; Costa, L. F. S.; Silva, M. A. A. (2010) "Aprendendo a Ensinar Programação Combinando Jogos e Python". In: XXI Simpósio Brasileiro de Informática na Educação - SBIE.

Sá, E. J. V; Teixeira, J. S. F; Fernandes, C. T. (2007) "Design de atividades de aprendizagem que usam Jogos como princípio para Cooperação". In: XVIII Simpósio Brasileiro de Informática na Educação - SBIE.

Tang, Stephen Kelang, Hanneghan, Martin (2008) “ Towards a Domain Specific Modelling Language for Serious Game Design" In: 6th International Game Design and Technology Workshop (GDTW'08). 